\title{
THE BACTERICIDAL ACTION OF ULTRA-VIOLET LIGHT
}

\author{
By D. E. LEA, M.A., Ph.D. and R. B. HAINES, D.Sc., Ph.D. \\ Strangeways Research Laboratory and \\ Low Temperature Research Station, Cambridge
}

(With 4 Figures in the Text)

\section{INTRODUCTION}

IT is well known that ultra-violet light exerts a strong bactericidal action at wave-lengths shorter than $3000 \mathrm{~A}$., and a number of authors have made quantitative investigations of the effect of varying the wave-length and the doses of radiation used. The experiments of several workers (Gates, 1930; Wyckoff, 1932; Herćik, 1936; Duggar \& Hollaender, 1934) agree in indicating that the effectiveness of the radiation per unit of energy increases as the wave-length is reduced below $3000 \mathrm{~A}$., and reaches a maximum between 2500 and $2800 \mathrm{~A}$. It is also agreed that this result holds for all species of bacteria investigated and is true whether the energy incident on the bacteria (in ergs $/ \mathrm{cm} .{ }^{2}$ ) or the energy absorbed by the bacterium (in ergs $/ \mathrm{cm} .{ }^{3}$ ) is measured. The principal facts concerning the variation of bactericidal effect with wavelength are thus known, and in the work described in this paper a single wavelength of radiation only has been used, namely $2537 \mathrm{~A}$., which lies near the peak of the curve of bactericidal efficiency. Using this radiation we have investigated two points on which agreement has not been reached in the work of previous observers, namely, the shape of the curve obtained by plotting the surviving fraction of organisms against the dose administered, and the effect upon the rate of death of varying the intensity of the radiation.

Wyckoff (1932), Hollaender \& Claus (1936), Heřcik (1936) and others have obtained exponential survival curves, i.e. when the logarithms of the proportions of bacteria surviving given doses of radiation are plotted against those doses straight lines are obtained, indicating that the death-rate is uniform throughout the exposure. Other observers, notably Coblentz \& Fulton (1924), Baker \& Nanavutty (1929), Gates $(1929,1930)$ and Duggar \& Hollaender (1934), have obtained sigmoid curves, indicating that the death-rate is initially low, rising later in the exposure. Our observations indicate that the survival curves are exponential.

The effect upon the death-rate of varying the intensity of the radiations has not so far been studied much. Gates (1929) found that the death-rate of Staphylococcus aureus irradiated by a wave-length of 2660 A. was not exactly proportional to the intensity, so that a given dose was more effective when 
delivered at high intensity than when delivered at low intensity. Bat the effect found was very small and it seems desirable to use a much greater variation of intensity than the fourfold variation used by Gates. Coblentz \& Fulton (1924) used a fiftyfold variation of intensity and reported a small difference, again in the direction of the more intense radiation being more effective for equal doses of radiation. They found, however, that dividing a given dose into a number of fractions with periods of rest between did not reduce its effectiveness. Dushkin \& Bachem (1933), who used a sixtyfold variation of intensity, failed to find any difference, though it may be doubted whether their experimental arrangement would detect any but a large difference. In the experiments to be described we have used an intensity variation of five hundred times, and have not been able to detect any variation of lethal dose with intensity, i.e. the death-rate is accurately proportional to the intensity over this range.

\section{Experimental methods AND Results}

In our experiments aqueous suspensions of the bacteria, exposed to the radiation in quartz tubes, were diluted and plated on nutrient agar in the usual fashion immediately after irradiation. This procedure seems to be preferable to the method which has often been employed of spreading a bacterial suspension uniformly over an agar plate, irradiating selected areas of the plate, and comparing the numbers of colonies forming on the various areas when the plate is incubated. In this last method the bacteria remain in contact with a nutrient medium which has been irradiated and which may have suffered deleterious chemical change in consequence. It is true that some authors have taken the precaution (e.g. Coblentz \& Fulton, 1924) of sowing an irradiated plate with unirradiated bacteria and have shown that the growth of the bacteria is not affected unless the agar is irradiated with higher doses than suffice to kill the bacteria when they themselves are irradiated. But this test does not exclude the possibility of the production of an unstable chemical disinfectant in the nutrient agar capable of acting upon bacteria present at the time of irradiating, but disappearing in the interval between irradiation and sowing of the plates in control experiments. There is the further objection that the sensitivity of the bacteria to radiation is liable to change when they are left in the presence of a nutrient for prolonged periods, and this is liable to lead to systematic error when the effect of equal doses administered in unequal times are being compared. The existence of a disturbing effect of the latter kind was demonstrated by Dushkin \& Bachem (1933).

We find that bacteria in suspension in glass distilled water, or better, in tap water, remain for some hours with only a slight fall in the viable count, and the absorption of ultra-violet light by the suspension and the quartz tube is small and readily allowed for. The bacteria were prepared by emulsifying the growth taken off an $18 \mathrm{hr}$. agar slant and shaking the suspension vigorously for about $10 \mathrm{~min}$. It was essential for the present work to make sure that the bacteria were present in the suspension as single cells and not as clumps, as 
the existence of an appreciable proportion of such clumps leads to the distortion of the shape of the survival curve in the direction of introducing a spurious initial flat portion to the curve. The existence of clumps among the bacteria used probably accounts for the sigmoid survival curves found by certain of the authors quoted in the previous section. A convenient and efficient shaker suitable for dealing with quantities up to about $20 \mathrm{ml}$. may be made by strengthening the cone of a moving coil loud-speaker with a linen reinforcement gummed on to it and attaching a holder for the tube of suspension. The speech coil is fed with A.c. from the mains (via the step-down transformer supplied with the speaker) and sets the bacterial suspension into rapid vibration, the amplitude of which may be regulated as desired by a resistance in the mains lead. The satisfactory dispersal of the bacteria into single organisms was checked by microscopic observation under dark field illumination. Suspensions containing from $10^{5}$ to $10^{7}$ organisms per ml. were employed, from which serial dilutions were made to obtain a convenient number of organisms on the plates. The source of ultra-violet light was a mercury lamp (Vitreosil "home-sun") of the type containing neon, operating at a temperature of about $50^{\circ} \mathrm{C}$., and from which $95 \%$ of the energy radiated is in the resonance line $2537 \mathrm{~A}$. The lamp consumed about $50 \mathrm{~W}$. at $500 \mathrm{~V}$. A.c. supplied by a shunted-core transformer and at 1 metre distance gave an ultra-violet intensity of approximately $500 \mathrm{ergs} / \mathrm{cm} .2 / \mathrm{sec}$. The radiation from this lamp being nearly monochromatic and of a wave-length near the peak of the bactericidal band is very suitable for experiments of this sort. In measuring the intensity a device had to be adopted capable of recording the ultra-violet intensity in the presence of a certain amount of visible light. For this purpose a method similar in principle to that used by Coblentz \& Stair (1936) to measure the ultra-violet light in sunlight in the presence of visible light was adopted. A thermopile was constructed of gauge 42 copper and constantin wires, having nine pairs of junctions sealed between two sheets of thin blackened paper. The thermopile was built into a thick-walled brass box, the hot and cold junctions being about $1.5 \mathrm{~cm}$. apart. Two apertures $1 \mathrm{~cm}$. diameter in the lid of the box gave access to the hot and cold junctions respectively; the former aperture was covered by quartz and the latter by glass. These windows were thick enough (3 mm.) to cut off the small radiant heat emission of the lamp and were equally transparent to visible light. The instrument was used by observing the difference in the e.m.f.'s generated in the presence and in the absence of a sheet of glass which could be interposed between the lamp and the thermopile. In the presence of this sheet of glass, which absorbed the ultra-violet light from the lamp, the two receivers were equally affected. When it was removed the receiver under the quartz window was illuminated by ultra-violet light in addition to the visible light which continued to be equal for the two receivers. The e.m.f. difference recorded was thus a measure of the ultra-violet light intensity. The sensitivity of the instrument was measured by completely obscuring one receiver and exposing the other (with no window over the 
aperture) to black body heat radiation; it was $3.0 \times 10^{-9} \mathrm{~V}$. for $1 \mathrm{erg} / \mathrm{cm} .{ }^{2} / \mathrm{sec}$. incident energy, and was measured by a low resistance suspended coil galvanometer. Full deflexion required about $40 \mathrm{sec}$. As the lowest intensities used in this work (120 ergs $/ \mathrm{cm}^{2} / \mathrm{sec}$.) gave too small deflexions on the galvanometer available, the intensities were calculated from the measured intensities obtained at shorter distances between lamp and thermopile. It can readily be shown that the energy flux on a small flat receiver at distance $r$ from a lamp consisting

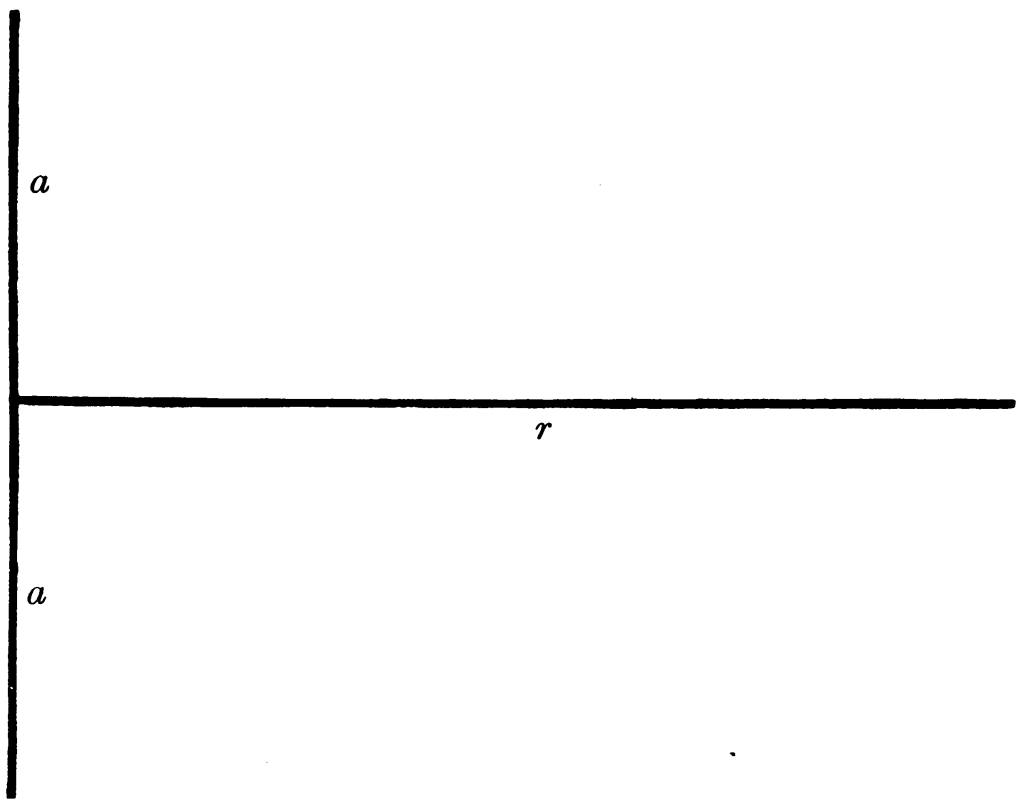

Fig. 1 (see text). $2 a$ =length of lamp; $r=$ distance of receiver from lamp.

of a narrow tube of length $2 a$ (see Fig. 1) emitting equally in all directions according to the inverse square law is proportional to

$$
\frac{1}{r^{2}}\left(1+a^{2} / r^{2}\right)^{-\frac{1}{2}} \text {. }
$$

The energy absorbed by a tube of bacteria placed at the same position as the receiver is proportional to a slightly different expression

$$
\frac{1}{r^{2}} \frac{\tan ^{-1}(a / r)}{(a / r)},
$$

the difference being due to the fact that the response of the thermopile varies with the direction of the radiation while the biological response does not. Formula (1) was verified by experiment at several distances and formula (2) was used to calculate the radiation intensity at the position occupied by the bacteria. The experiment was performed in a room with blackened walls and ceiling to avoid scattering of light which would make the inverse square law invalid. At the highest intensity used (at a distance of 4-5 cm. from the lamp) the exposures required ranged from one-tenth of a second upwards, and a 
mechanical device was needed to make these short exposures. For this purpose a slit of adjustable width was mounted on a gramophone turntable driven by an electric motor at a speed of $1 \mathrm{rev} . / \mathrm{sec}$. With this arrangement a slit of width $1 \mathrm{~cm}$. gave an exposure of $0.01 \mathrm{sec}$, and the required short exposures could be given with sufficient accuracy by suitable choice of slit width.

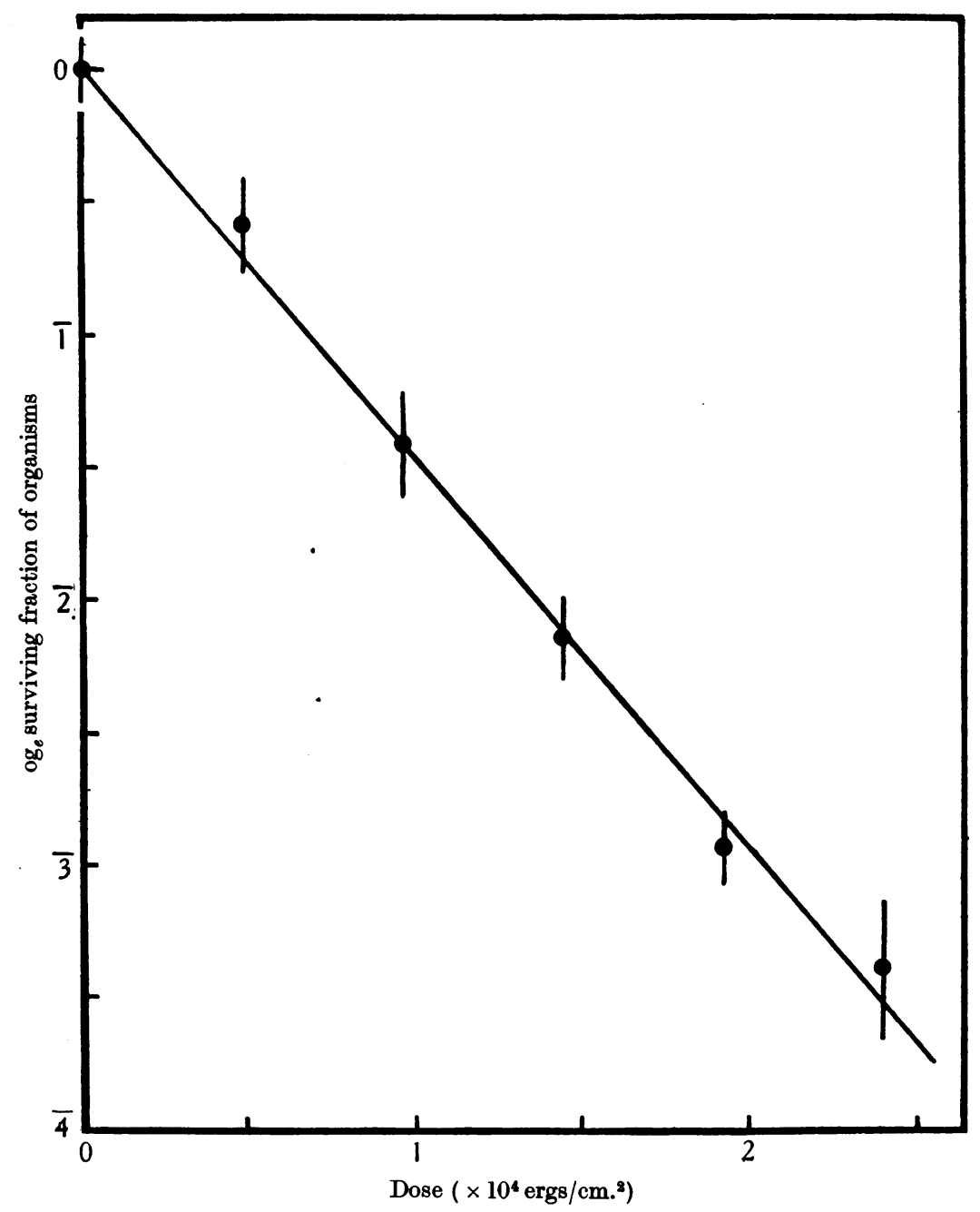

Fig. 2. Action of ultra-violet light (intensity $120 \mathrm{ergs} / \mathrm{cm} .{ }^{2} / \mathrm{sec}$.) on aqueous suspensions of Bact. prodigiosum.

The results obtained are summarized in Figs. 2-4, in which the natural logarithms of the fractions of the initial numbers of bacteria surviving irradiation are plotted against the doses administered (dose $=$ intensity $\times$ time). All the survival curves are exponential within the random error of the experimental determination. In the case of Bact. prodigiosum [Serratia marcesens] (Fig. 2) 
the determination of the survival curve was carried through six times, every sample being plated in triplicate. The probable errors shown are calculated from the mutual consistency of the repeated results. In the case of the other organisms (Figs. 3, 4) fewer repetitions were employed, but such deviations as occur from the expected survival curve appear to be random rather than systematic. The variations appear to be greater in the lower halves of the survival curves in Figs. 3 and 4 than in the upper halves; but it should be

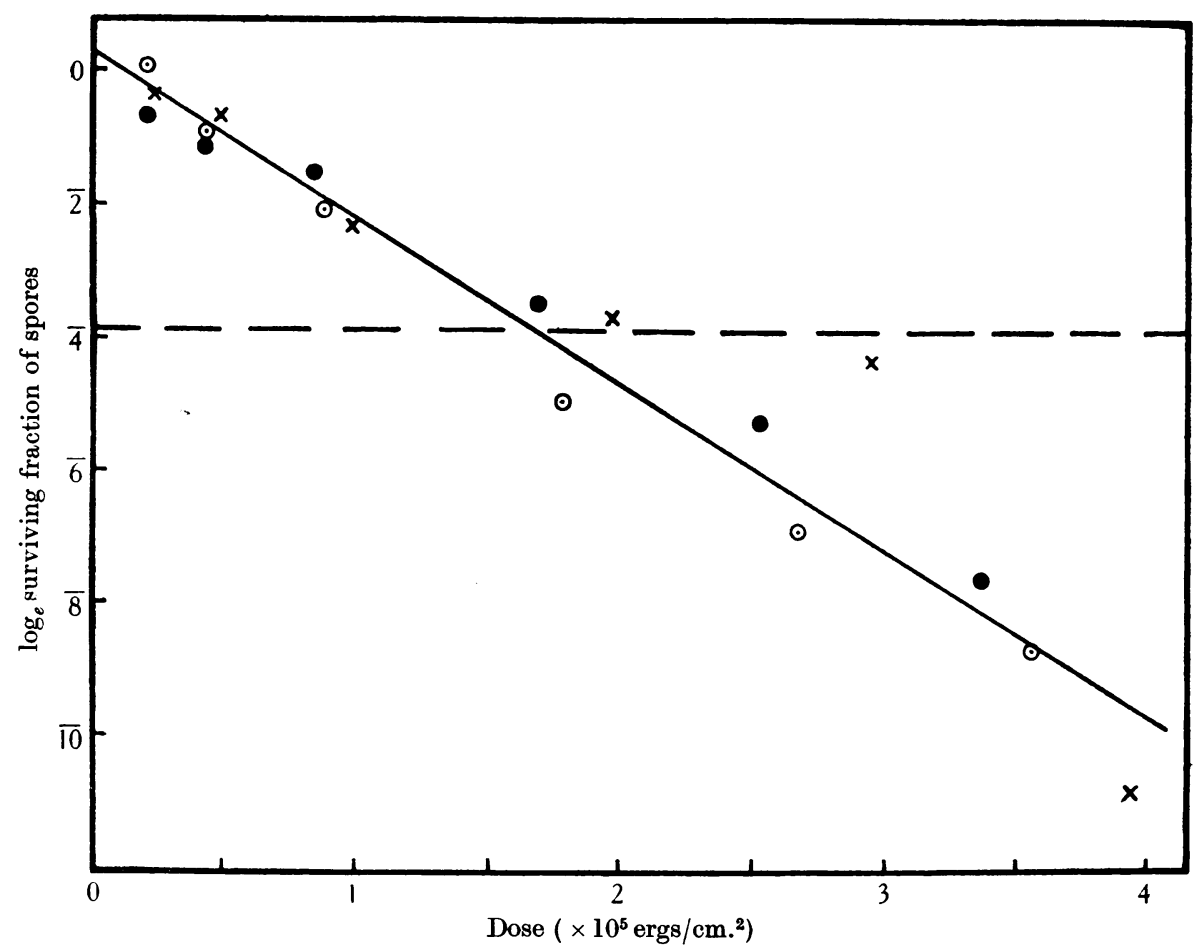

Fig. 3. Action of various intensities of ultra-violet light on spores of B. mesentericus. $7 \cdot 0 \times 10^{4}$ ergs $/ \mathrm{cm} .{ }^{2} / \mathrm{sec} . \times 4.1 \times 10^{3} \mathrm{ergs} / \mathrm{cm}^{2} / \mathrm{sec}$. $\odot 1.48 \times 10^{2} \mathrm{ergs} / \mathrm{cm} .{ }^{2} / \mathrm{sec}$. The portion of the curve below the broken line refers to the last $2 \%$ of the spores.

pointed out that the lower halves relate to the killing only of the last $1 \%$ of the organisms. The method of plotting the surviving numbers on a logarithmic scale exaggerates the errors of the points in the second half of the curve; expressed as fractions of the initial number of bacteria they would be insignificant.

In Figs. 3 and 4 the points referring to three different intensities covering a range of 500 to 1 are shown together and evidently are satisfactorily fitted by the same curve indicating that the rates of death are accurately proportional to the intensities. 


\section{Discussion}

It is of interest to compare the amount of ultra-violet light energy required to kill a bacterium with the amount of energy of other radiations. It can readily be shown that when the survival curves are exponential the mean dose required

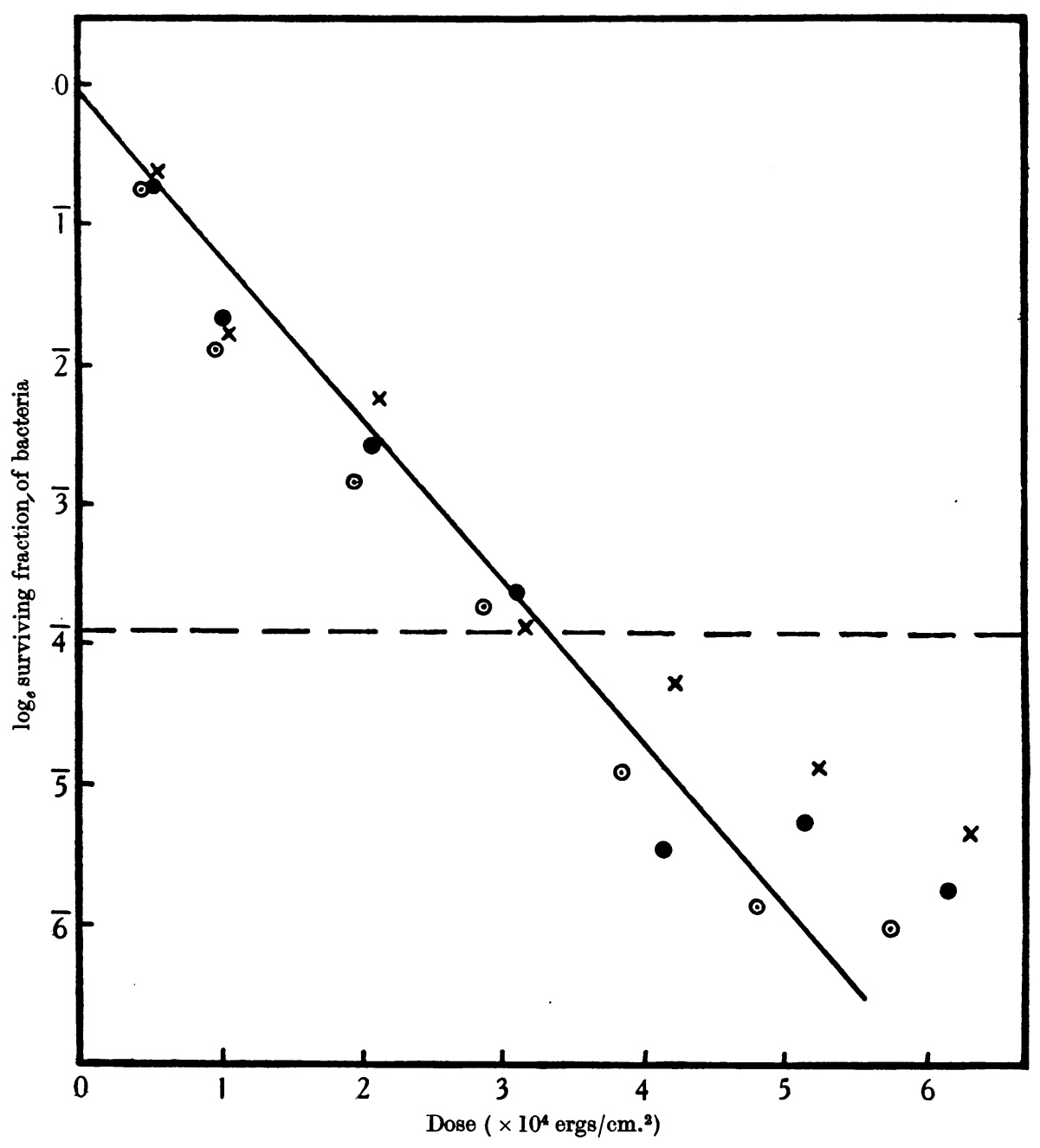

Fig. 4. Action of various intensities of ultra-violet light on Bact. coli. $6.43 \times 10^{4} \mathrm{ergs} / \mathrm{cm}^{2} / \mathrm{sec}$. $\times 3.1 \times 10^{3} \mathrm{ergs} / \mathrm{cm}^{2} / \mathrm{sec}$. $\odot 1.2 \times 10^{2} \mathrm{ergs} / \mathrm{cm}^{2} / \mathrm{sec}$. The portion of the curve below the broken line refers to the last $2 \%$ of the bacteria.

to kill a bacterium is that dose which kills $64 \%$ of the bacteria, i.e. which reduces the surviving fraction to $\bar{I}$ on the logarithmic scale of Figs. $2-4$. The mean lethal doses are given in Table 1 (first column) in terms of energy incident on the bacteria; to calculate the energy absorbed the absorption coefficient in the part of the bacterium concerned in the lethal effect must be known. 
Table 1. Comparison of lethal doses of bacteria irradiated by ultra-violet light and by $X$-rays

\begin{tabular}{|c|c|c|c|c|c|}
\hline \multirow{3}{*}{ Organism } & \multicolumn{2}{|c|}{$\begin{array}{l}\text { Mean lethal dose to } \\
\text { ultra-violet (2537 A.) }\end{array}$} & \multicolumn{2}{|c|}{$\begin{array}{c}\text { Mean lethal dose to } \\
\text { X-rays (1.5 A.) }\end{array}$} & \multirow{2}{*}{$\begin{array}{c}\text { Ratio } \\
\text { quanta : ions }\end{array}$} \\
\hline & Ergs/cm. ${ }^{2}$ & Quanta/cm. ${ }^{3}$ & $r$-units & Ions $/ \mathrm{cm}^{3}{ }^{3}$ & \\
\hline & 1 & 2 & 3 & 4 & 5 \\
\hline Bact. prodigiosum & $6 \cdot 8 \times 10^{3}$ & $3 \cdot 17 \times 10^{18}$ & $3.5 \times 10^{3}$ & $4.55 \times 10^{15}$ & 697 \\
\hline Bact. coli & $8.6 \times 10^{3}$ & $4.01 \times 10^{18}$ & $6.5 \times 10^{3}$ & $8.45 \times 10^{15}$ & 475 \\
\hline $\begin{array}{l}\text { Spores of } \\
\text { B. mesentericus }\end{array}$ & $4.0 \times 10^{4}$ & $1.86 \times 10^{19}$ & $1.3 \times 10^{5}$ & $1.69 \times 10^{17}$ & 110 \\
\hline
\end{tabular}

This is not known, but Gates (1930) has measured the absorption coefficient of bacterial protoplasm as a whole and finds it to be $3.6 \times 10^{3} \mathrm{~cm} .^{-1}$ for wavelength $2537 \mathrm{~A}$. The mean lethal dose for Bact. prodigiosum, $6.8 \times 10^{3} \mathrm{ergs} / \mathrm{cm}^{2}$ incident energy, thus becomes $6.8 \times 10^{3} \times 3.6 \times 10^{3}=2.45 \times 10^{7} \mathrm{ergs} / \mathrm{cm} .^{2} \mathrm{ab}-$ sorbed energy. Now the quantum of ultra-violet light of wave-length $2537 \mathrm{~A}$. has energy $7 \cdot 74 \times 10^{-12}$ ergs. Thus the mean lethal dose corresponds to the production of $2.45 \times 10^{7} \div 7.74 \times 10^{-12}=3.17 \times 10^{18}$ quanta per $\mathrm{cm}^{3}$ in the bacterium. The corresponding figures for the other bacteria used in these experiments, calculated in similar fashion, are listed in the second column of Table 1.

The lethal doses to X-rays, expressed in terms of $r$-units in column 3 , and of ionizations per cm. ${ }^{3}$ of tissue in column 4 , are also given (Lea \& Haines, in the Press). The order of sensitivity of these bacteria to ultra-violet light is the same as to X-rays. The number of absorbed quanta of ultra-violet equivalent in biological effect to one ionization is shown in column 5. The variation between the ratio found for the spores and the vegetative cells may possibly not be real but be due to the incorrect assumption of equal ultra-violet absorption coefficient for spores and vegetative cells; the spores probably have a larger absorption coefficient (cp. Herćik, 1936). In biological effect one ionization is equivalent to some hundreds of ultra-violet quanta. The energy associated with the production of an ionization is, however, only about six times the energy of an ultra-violet quantum. It is evident therefore that about a hundred times more energy is required to kill the bacterium administered as ultra-violet light than as ionization. Presumably in both cases the radiation produces its biological effect by the intermediary of chemical change. The differences in the efficiency may be interpreted to mean that different chemical reactions are involved in the two cases, the presumption being that the chemical reaction induced by the $\mathrm{X}$-rays cannot be induced by the ultra-violet light. This interpretation is, however, not at all an inevitable one. Firstly, it is possible that the ultra-violet absorption of the cell constituents taking part in the reaction may be less than the average for the bacterial protoplasm as a whole, so that the number of quanta absorbed per $\mathrm{cm}^{3}$ in this constituent may be less than calculated in Table 1. More plausible, however, is the suggestion 
that the quantum yields may be very small, of the order $0 \cdot 01-0 \cdot 001$. The energy involved in ionization is so considerable (on a molecular scale) that a molecule ionized by $\mathrm{X}$-rays is unlikely to survive the experience without chemical change. On the other hand the energy of an ultra-violet light quantum is not usually sufficient to cause ionization (cp. Crowther, 1938) and there are numerous instances where the quantum yield in a photochemical reaction (number of molecules transformed per quantum absorbed) is very small.

According to our interpretation (Lea \& Haines, in the Press) the action of $\mathrm{X}$-rays and other ionizing radiations on bacteria is to be thought of as a lethal mutation involving a single "gene" molecule without general destruction of the cell. It is presumed that a single ionization suffices to cause a mutation. It is evident from the present experiments that a single ultra-violet quantum absorbed by the gene has only a small probability of producing a mutation. This is not because the energy of the ultra-violet quantum is insufficient; it is unlikely that the molecule in question should be so stable as to require an activation energy of more than $4.9 \mathrm{~V}$. (which is provided by the quantum), but because when the molecule is excited by the absorption of a quantum it has nearly a $100 \%$ chance of disposing of its energy of excitation without chemical change.

\section{Summary}

Experiments on the bactericidal action of ultra-violet light have been made to determine the shape of the survival curve and the dependence upon radiation intensity of the rate of death. Bact. coli, Bact. prodigiosum and spores of B. mesentericus were irradiated with approximately monochromatic light of wave-length $2537 \mathrm{~A}$. The survival curves obtained were exponential and the rate of death was accurately proportional to the intensity over an intensity range of $500: 1$.

By comparing these results with data previously obtained of the action of $\mathrm{X}$-rays on the same organisms it was established that one ionization produced by $\mathrm{X}$-rays is as effective as some hundreds of ultra-violet quanta. This is interpreted to mean that the quantum yield in whatever chemical change leads to the loss of viability in the irradiated bacteria is, for $2537 \mathrm{~A}$., between 0.01 and $0 \cdot 001$.

\section{REFERENCES}

Baker, S. L. \& NanavutTy, S. H. (1929). A quantitative study of the effect of ultra-violet rays on the bacteriophage. Brit. J. exp. Path. 10, 45-61.

Coblentz, W. W. \& Fulton, H. R. (1924). A radiometric investigation of the germicidal action of ultra-violet radiation. Sci. Pap. Bur. Stand. no. 495, 19, 641-80.

Coblentz, W. W. \& StaIR, R. (1936). Evaluation of ultra-violet solar radiation of short wave-lengths. Bur. Stand. J. Res. 16, 315-47.

Crowther, J. A. (1938). The biological action of X-rays-a theoretical review. Brit. J. Radiol. 11, 132-45.

DUGGaR, B. M. \& HollaENDER, A. (1934). Irradiation of plant viruses and of microorganisms with monochromatic light. I and II. J. Bact. 27, 219-39, 241-56. 
Dushin, M. A. \& BaChem, A. (1933). Time factors concerned in the germicidal action of ultra-violet light. Proc. Soc. Exp. Biol., N.Y., 30, 700-4.

Gates, F. L. (1929). A study of the bactericidal action of ultra-violet light. I and II. J. gen. Physiol. 13, 231-48, 249-60.

Gates, F. L. (1930). A study of the bactericidal action of ultra-violet light. III. J. gen. Physiol. 14, 31-42.

HeŘććc, F. (1936). Action of ultra-violet light on spores and vegetative forms of $B$. megatherium sp. J. gen. Physiol. 20, 589-94.

Hollaender, A. \& Clads, W. D. (1936). The bactericidal effect of ultra-violet radiation on Escherichia coli in liquid suspensions. J. gen. Physiol. 19, 753-65.

HollaEnder, A. \& DugGar, B. M. (1936). Irradiation of plant viruses and of microorganisms with monochromatic light. III. Proc. Nat. Acad. Sci. 22, 19-24.

LEA, D. E. \& Haines, R. B. I. The lethal action of soft X-rays on bacteria. II. The mechanism of the bactericidal action of radiation. Z. Bakt. (in the Press).

Wyckoff, R. G. (1932). The killing of colon bacilli by ultra-violet light. J. gen. Physiol. 15, 341-61.

(MS. received for publication 14. XII. 39-Ed.) 